\title{
MÓDULO EM ESPACO DE ESTADOS PARA APLICAÇÃO DE TÉCNICAS DE CONTROLE PARA PLANTAS DE SEGUNDA ORDEM
}

João Victor Moura Silva - victormourasilval@gmail.com

Universidade Federal do Pará - UFPA

R. Augusto Corrêa, 01

66075110 - Belém - Pará

Itamarda Silva Pinto - itamarsp11@gmail.com

Arthur Felipe dos Santos Fernandes - arthurengl.ufpa@gmail.com

Lucas Manoel Moraes Portal-lucasmano230@gmail.com

Orlando Fonseca Silva-orfosi@ufpa.br

Resumo: O trabalho apresenta o projeto, a implementação e a aplicação de um módulo em espaço de estados para plantas de segunda ordem, com a sua utilização voltada aos discentes como forma de ajuda-los a melhor compreensão do tema. Foram utilizados componentes simples e de baixo valor visando a fácil reprodução, e, por conseguinte, a democratização da ferramenta. Isso posto, espera-se que a ferramenta contribua para a melhoria no ensino na engenharia, sobretudo as disciplinas voltadas a área de controle de sistemas dinâmicos.

Palavras-chave: Modulo Didático. Ensino Aprendizagem. Controle. Espaço de Estados.

\section{INTRODUÇÃO}

A técnica clássica para representação de modelos é baseada no domínio da frequência, a qual relaciona matematicamente a entrada e a saída de um sistema por meio de sua função de transferência. A aplicação de funções de transferência torna-se complicada para sistemas com múltiplas entradas e saídas (MIMO, multiple-input and multiple-output), sistemas variantes no tempo ou sistemas não-lineares. Nestes casos é mais conveniente o uso da teoria de controle moderno por espaço de estados (OGATA, 1985).

A abordagem pelo espaço de estados é a mais apropriada para analisar sistemas modernos complexos com várias entradas e saídas (OGATA, 1985). Isso posto, evidencia-se a importância da plena compreensão do funcionamento, análise e síntese de sistemas dinâmicos, sobretudo, para os discentes das engenharias.

Com base nisso, desenvolveu-se um módulo didático, construído com componentes eletrônicos de fácil aquisição, capaz de emular uma planta de segunda ordem, com seus parâmetros dimensionados para uma melhor resposta às entradas e aquisição de dados pelo usuário. Outra característica do protótipo, é a possibilidade de monitorar os dados do sistema objetivando a realização dos ajustes necessários em tempo real para que o módulo didático funcione adequadamente no decorrer do tempo, para essa tarefa utiliza-se uma placa Arduino.

O desenvolvimento deste módulo tem objetivo de proporcionar aos discentes dos cursos de Engenharia a experiência no uso da teoria de espaço de estados aplicada na análise e controle de sistemas dinâmicos, a fim de consolidar o conhecimento teórico adquirido nas 
(C) COBENGE

"Os desafios para formar hoje o engenheiro do amanhã"
$\mathrm{Ol} \mathrm{a} \mathrm{O3}$ de dezembro Evento On-line

disciplinas de controle. Além disso, uma vez concluído, o projeto proporcionará uma estratégia didática alternativa às simulações via softwares computacionais, com o objetivo de aumentar o interesse dos discentes e aproximando-os da realidade prática nesta área de conhecimento.

\section{MATERIAIS E MÉTODOS}

A Figura 1 ilustra o diagrama de simulação da planta a ser controlada. Este diagrama será implementado em um circuito elétrico com amplificadores operacionais.

Figura 1 - Diagrama de simulação da planta

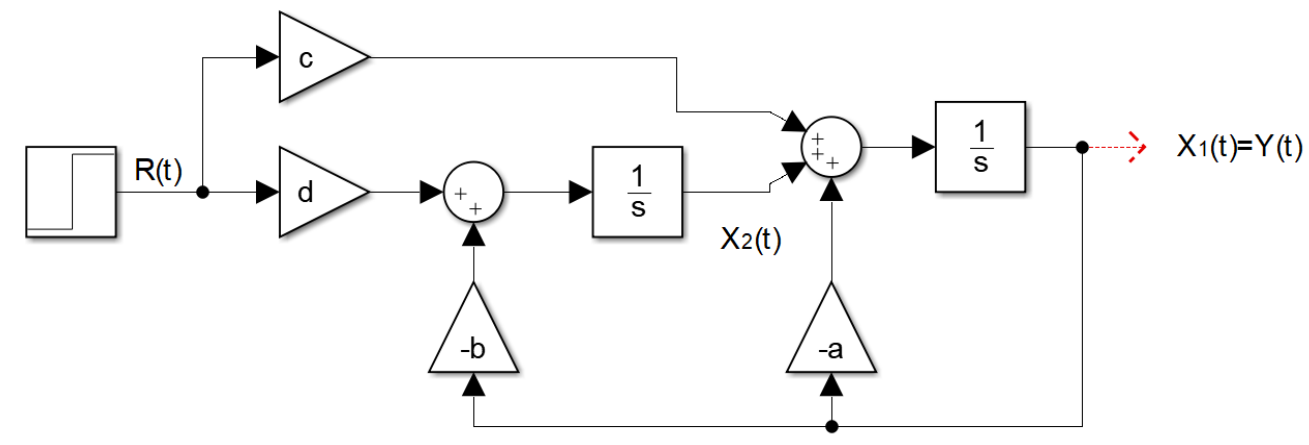

Fonte: Autores.

Uma representação no espaço de estados e equação de saída para o diagrama da Figura 1, esta é apresentada nas Equações (1) e (2), e sua correspondente função de transferência na Equação (3).

$$
\begin{gathered}
\dot{\mathrm{X}}(\mathrm{t})=\left[\begin{array}{c}
\dot{x}_{1}(\mathrm{t}) \\
\dot{x}_{2}(\mathrm{t})
\end{array}\right]=\left[\begin{array}{cc}
-a & 1 \\
-b & 0
\end{array}\right] \mathrm{X}(\mathrm{t})+\left[\begin{array}{l}
c \\
d
\end{array}\right] \mathrm{u}(\mathrm{t})=\mathrm{AX}(\mathrm{t})+\mathrm{B} \mathrm{u}(\mathrm{t}) \\
y(\mathrm{t})=\mathrm{C} \mathrm{X}(\mathrm{t})=\left[\begin{array}{cc}
1 & 0
\end{array}\right] \mathrm{X}(\mathrm{t}) \\
\mathrm{G}(\mathrm{S})=\frac{c \mathrm{~S}+d}{\mathrm{~S}^{2}+a \mathrm{~S}+b}=\frac{\mathrm{Y}(\mathrm{S})}{\mathrm{U}(\mathrm{S})}
\end{gathered}
$$

Observa-se que a função de transferência genérica de segunda ordem da planta, garante a existência de um zero real e sua representação no espaço de estados garante que a saída $y(\mathrm{t})$ é igual a variável de estado $x_{1}(\mathrm{t})$.

Para garantir a necessidade de um controlador, escolheu-se os seguintes valores numéricos: $a=1, b=0, c=1$ e $d=15$, que tornam o sistema em malha aberta BIBO instável, tendo como resposta ao degrau unitário o sinal mostrado na Figura 2.

Figura 2 - Resposta da planta ao degrau unitário. 


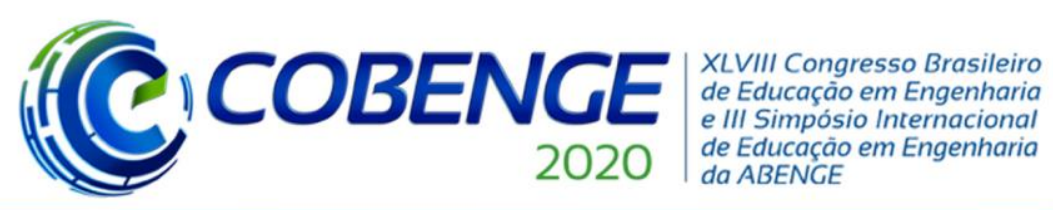

"Os desafios para formar hoje o engenheiro do amanhã"

\section{Evento On-line}

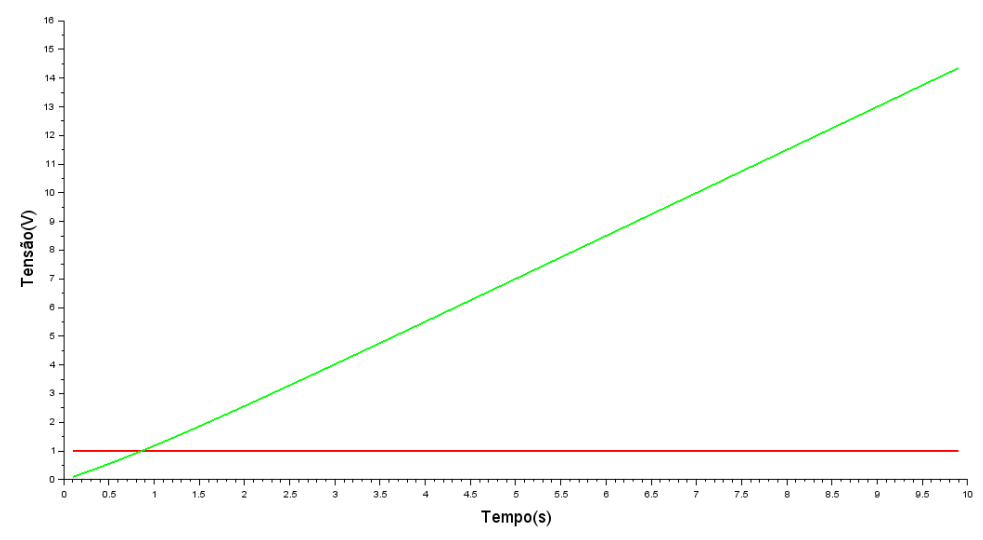

Fonte: Autores.

Após assegurar que a planta não tem um bom desempenho em malha aberta é possível pensar em alguma técnica para realizar o controle dessa planta.

Com os valores numéricos escolhidos para $a, b, c$ e $d$, resultam para sua função de transferência a Equação (4). Como o controlador a ser utilizado é digital, pois será implementado no Arduino, deve-se avaliar primeiramente qual o período de amostragem $T_{\mathrm{s}} \mathrm{a}$ ser utilizado. Na Equação (4) tem-se como constantes de tempo $\mathrm{T}_{1}=1 / 15$ e $\mathrm{T}_{2}=1$. Sendo a menor constante de tempo: $\mathrm{T}_{\min }=1 / 15$. Pela Inequação (5), resulta que o período de amostragem $\mathrm{T}_{\mathrm{S}}$ a ser adotado está compreendido no intervalo: $0.0066<=\mathrm{T}_{\mathrm{S}}<=0.0133$.

$$
\begin{aligned}
& \mathrm{G}(\mathrm{S})=\frac{\mathrm{S}+15}{\mathrm{~S}^{2}+\mathrm{S}}=\frac{15\left(\frac{\mathrm{s}}{15}+1\right)}{s(s+1)} \\
& \mathrm{T}_{\min } / 10<=\mathrm{T}_{\mathrm{S}}<=\mathrm{T}_{\min } / 5
\end{aligned}
$$

Arredondando o maior valor possível do período de amostragem para $\mathrm{T}_{\mathrm{s}}=0.01$, e sendo este valor bastante pequeno, pode-se, numa primeira aproximação, considerar que o controlador é analógico e esta será a primeira abordagem para projeto do controlador. Em seguida o projeto será realizado considerando um controlador digital. Os resultados obtidos por simulação, nos dois casos, serão comparados com os resultados do sistema real.

A Figura 3 ilustra o sistema contínuo em malha fechada, sendo as realimentações das variáveis de estado $\mathrm{x}_{1}(\mathrm{t})$ e $\mathrm{x}_{2}(\mathrm{t})$ ponderadas pelos ganhos $\mathrm{k}_{1} \mathrm{e} \mathrm{k}$ e a referência pelo ganho $\propto$.

Figura 3 - Planta do tipo 1 com o controle por alocação de polos.

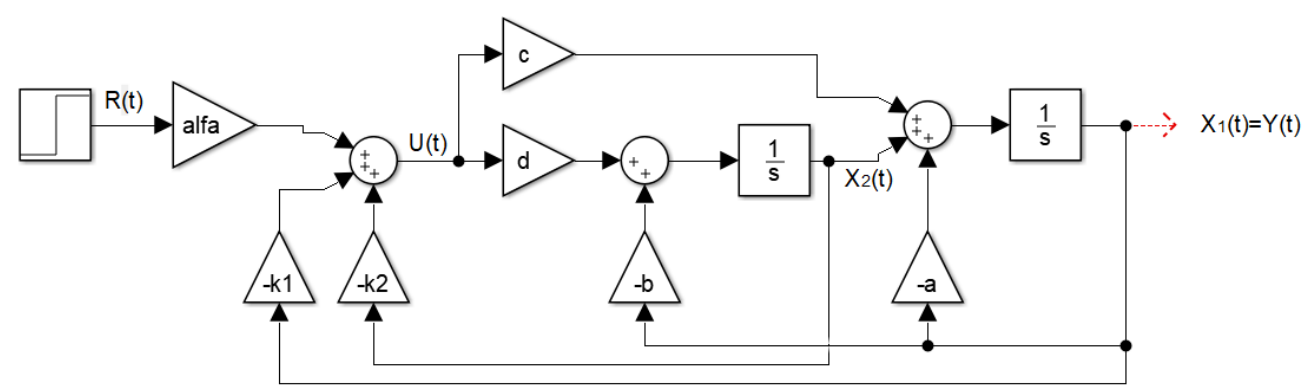

Fonte: Autores. 
(C) COBENCE

"Os desafios para formar hoje o engenheiro do amanhã"
$\mathrm{Ol} \mathrm{a} \mathrm{O} 3$ de dezembro

\section{Evento On-line}

Assim, a ação de controle é representada pela Equação (6), substituindo-a na Equação (1), resulta que o sistema em malha fechada é dado pela Equação (7), cuja função de transferência correspondente é dada pela Equação (8).

$$
\begin{gathered}
\mathrm{u}(\mathrm{t})=-k_{1} x_{1}(t)-k_{2} x_{2}(t)+\alpha \mathrm{r}(\mathrm{t})=-\mathrm{KX}(\mathrm{t})+\alpha \mathrm{r}(\mathrm{t})=; \forall \alpha \in \mathbb{R} \\
\dot{\mathrm{x}}(\mathrm{t})=\mathrm{Ax}(\mathrm{t})+\mathrm{B}\left[-K_{c} \mathrm{x}(\mathrm{t})+\alpha \mathrm{r}(\mathrm{t})\right]=\left[\mathrm{A}-\mathrm{B} K_{c}\right] \mathrm{x}(\mathrm{t})+\mathrm{B} \alpha \mathrm{r}(\mathrm{t}) ; \\
G_{m f}=\frac{Y(s)}{R(s)}=\mathrm{C}\left[\mathrm{sI}-\left(\mathrm{A}-\mathrm{B} K_{c}\right)\right]^{-1} \mathrm{~B} \alpha
\end{gathered}
$$

O problema de projeto por realimentação de estados consiste em determinar o vetor de ganhos $K_{c}$ tal que o sistema em malha fechada tenha um conjunto de polos previamente especificados. Sendo a planta de ordem dois, este cálculo pode ser feito pelo uso da Equação (9) (Ackerman), sendo $\mathrm{Pd}_{\mathrm{d}}(\mathrm{A})$ obtido pela Equação (10), Pd(s) pela Equação (11) e $\lambda_{1}$ e $\lambda_{2}$ os polos desejados para o sistema em malha fechada.

$$
\begin{gathered}
K_{c}=\left[\begin{array}{ll}
0 & 1
\end{array}\right][\mathrm{B} \mathrm{AB}]^{-1} P_{d}(\mathrm{~A}) \\
P_{d}(\mathrm{~A})=\left.P_{d}(\mathrm{~s})\right|_{\mathrm{s}=\mathrm{A}} \\
P_{d}(\mathrm{~S})=\left(\mathrm{s}-\lambda_{1}\right)\left(\mathrm{s}-\lambda_{2}\right)
\end{gathered}
$$

Escolhendo para a malha fechada: $\lambda_{1}=\lambda_{2}=-1$, resulta: $K_{c}=\left[\begin{array}{ll}0 . & 0.0666667\end{array}\right]$. Substituindo o $K_{c}$ obtido na Equação (8), a função de transferência de malha fechada é dada pela Equação (12).

$$
G_{m f}=\frac{Y(s)}{R(s)}=\frac{s+15}{s^{2}+2 s+1} \propto
$$

Sendo a referência $\mathrm{R}(\mathrm{s})$, um degrau unitário, a resposta em regime permanente é dada pela Equação (13) (Teorema do Valor Final). Como se deseja erro nulo em regime então $\propto=1$ / 15.

$$
y(\infty)=15 \propto
$$

Para o caso discreto, na Figura 3 tem-se: $\mathrm{R}\left(\mathrm{kT}_{\mathrm{s}}\right), \mathrm{U}\left(\mathrm{kT}_{\mathrm{s}}\right), \mathrm{x}_{1}\left(\mathrm{kT}_{\mathrm{s}}\right), \mathrm{x}_{2}\left(\mathrm{kT}_{\mathrm{s}}\right)$ e os integradores (1/s) tornam-se atrasos no tempo $\left(\mathrm{z}^{-1}\right)$. Em malha aberta as Equações 1 e 2 passam a ser as Equações (14) e (15), sendo $\emptyset$ e $\gamma$ obtidos pelas Equações (16) e (17) e $T_{S}$ o período de amostragem.

$$
\begin{gathered}
\mathrm{X}(\mathrm{k}+1)=\left[\begin{array}{l}
x_{1}(k) \\
x_{2}(k)
\end{array}\right]=\emptyset \mathrm{X}(\mathrm{k})+\gamma \mathrm{u}(\mathrm{k}) \\
y(\mathrm{k})=\mathrm{C} \mathrm{X}(\mathrm{k})=\left[\begin{array}{ll}
1 & 0
\end{array}\right] \mathrm{X}(\mathrm{k}) \\
\varnothing=e^{-A T_{s}} \\
\gamma=\int_{0}^{T_{S}} e^{A \mu} \mathrm{Bd} \mu
\end{gathered}
$$

Em malha fechada as Equações (6), (7) e (8) passam a ser as Equações (18), (19) e (20).

$$
\begin{gathered}
\mathrm{u}(\mathrm{k})=-k_{1} x_{1}(k)-k_{2} x_{2}(k)+\alpha \mathrm{r}(\mathrm{k})=-K_{d} \mathrm{X}(\mathrm{k})+\alpha \mathrm{r}(\mathrm{k}), \forall \alpha \in \mathbb{R} \\
\mathrm{X}(\mathrm{k}+1)=\emptyset \mathrm{X}(\mathrm{k})+\gamma\left[-K_{d} \mathrm{x}(\mathrm{k})+\alpha \mathrm{r}(\mathrm{k})\right]=\left[\emptyset-\gamma K_{d}\right] \mathrm{x}(\mathrm{k})+\mathrm{B} \alpha \mathrm{r}(\mathrm{k}) ;
\end{gathered}
$$




\section{COBENGE (c) 2020

$$
G_{m f}=\frac{Y(z)}{R(z)}=\mathrm{C}\left[\mathrm{zI}-\left(\varnothing-\gamma K_{d}\right)\right]^{-1} \gamma \alpha
$$

Para o cálculo de $K_{d}$ as Equações (9), (10) e (11) são substituídas pelas Equações (21), (22) e (23), onde $z_{1}$ e z2 podem ser obtidos pelas Equações (24) e (25).

$$
\begin{gathered}
K_{d}=\left[\begin{array}{ll}
0 & 1
\end{array}\right][\gamma \emptyset \gamma]^{-1} P_{d}(\varnothing) \\
P_{d}(\varnothing)=\left.P_{d}(\mathrm{z})\right|_{\mathrm{z}=\varnothing} \\
P_{d}(\mathrm{z})=\left(\mathrm{z}-\mathrm{z}_{1}\right)\left(\mathrm{s}-\mathrm{z}_{2}\right) \\
\mathrm{z}_{1}=e^{\lambda_{1} T_{s}} \\
\mathrm{z}_{2}=e^{\lambda_{2} T_{s}}
\end{gathered}
$$

Como $\mathrm{T}_{\mathrm{s}}=0.01$, das Equações (16) e (17) têm-se as Equações (26) e (27) e como $\lambda_{1}=$ $\lambda_{2}=-1$ das equações (24) e (25) têm-se a Equação (28).

$$
\begin{gathered}
\varnothing=\left[\begin{array}{cc}
0.9900498 & 0.0099502 \\
0 & 1
\end{array}\right] \\
\gamma=\left[\begin{array}{c}
0.0106977 \\
0.15
\end{array}\right] \\
\mathrm{z}_{1}=\mathrm{z}_{2}=0.9900498
\end{gathered}
$$

Com as Equações (23), (22) e (21) resulta que $K_{d}=\left[\begin{array}{ll}-0.0000040 & 0.0663345\end{array}\right]$.

Substituindo o $K_{d}$ obtido na Equação (20), a função de transferência de malha fechada é dada pela Equação (29).

$$
G_{m f}=\frac{Y(z)}{R(z)}=\frac{0.0106977 z-0.0092051}{z^{2}-1.9800997 z s+0.98019871} \propto
$$

Sendo a referência $R(z)$ um degrau unitário, a resposta em regime permanente é dada pela Equação 30. Como se deseja erro nulo em regime então: $\propto=1 / 15.076768$.

$$
y(\infty)=15.076768 \propto
$$

A partir dos parâmetros obtidos $K_{c}, T_{s} e K_{d}$, os diagramas de simulação, para o caso contínuo e discreto, foram criados na ferramenta Xcos do Software Scilab, conforme as Figuras 4 e 5. Os respectivos resultados obtidos para a resposta ao degrau unitário são mostrados nas Figuras 6 e 7 .

Figura 4 - Diagrama de simulação da planta com controlador contínuo. 


\section{CORENGE (C) COBENCE 2020 e III Simpósio Internacional de Educação em Engenharia da ABENGE}

\section{"Os desafios para formar hoje o engenheiro do amanhã"}

\section{Evento On-line}

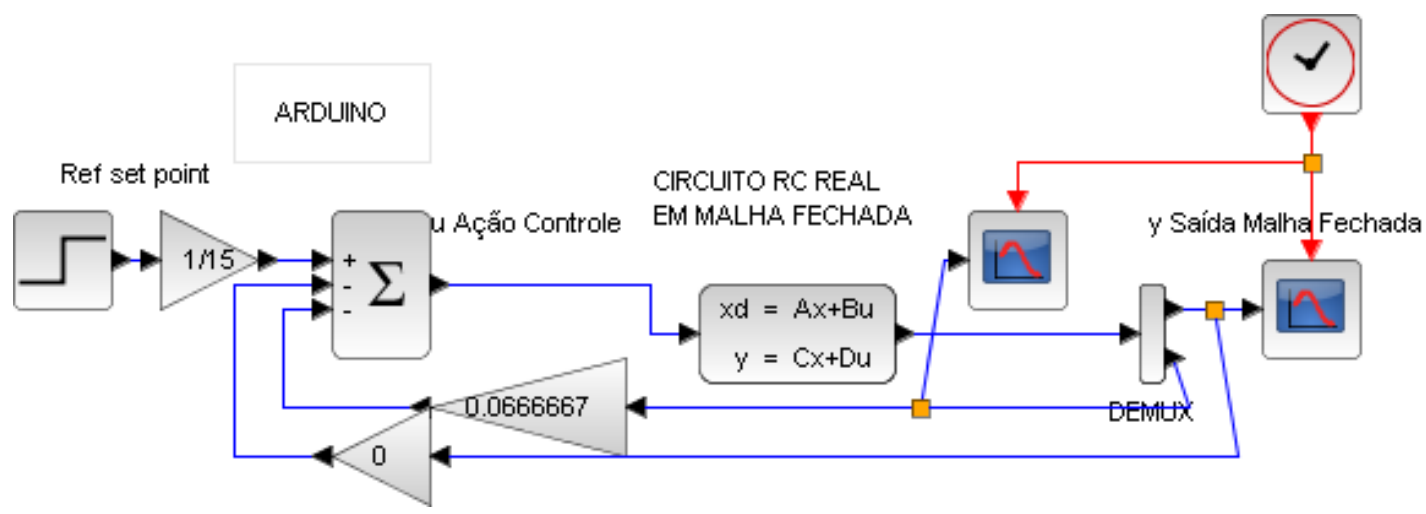

Fonte: Autores.

Figura 4 - Diagrama de simulação da planta com controlador discreto.

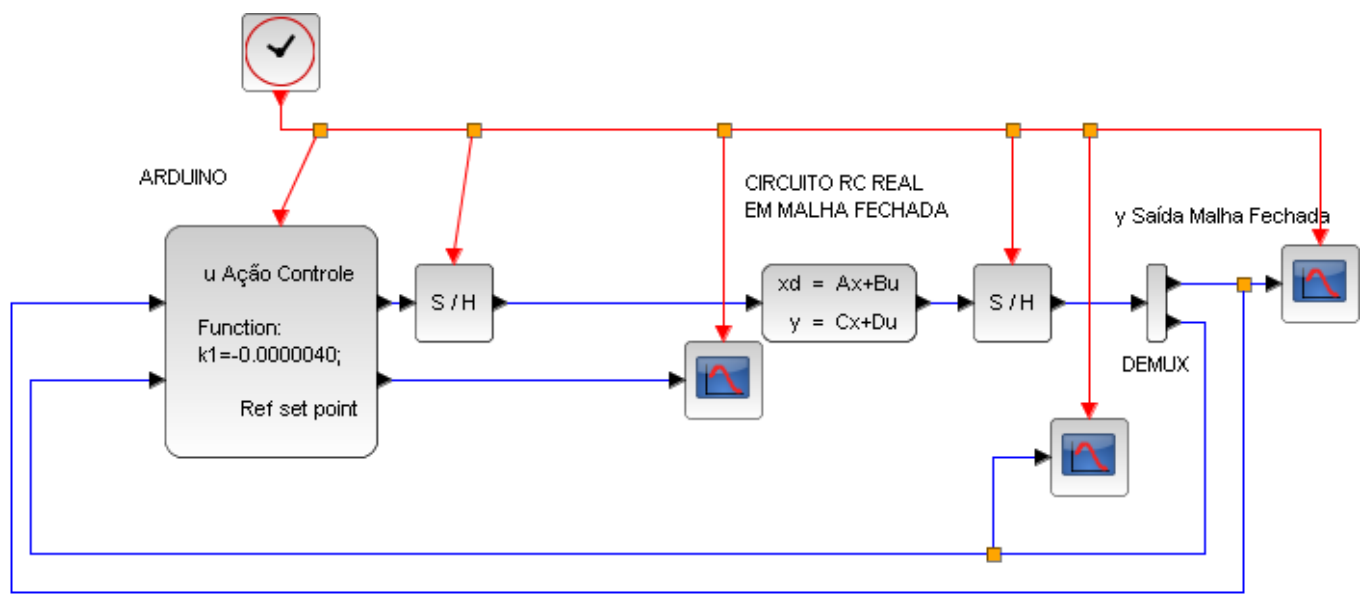

Fonte: Autores

Figura 6 - Resposta ao degrau unitário da planta com controlador contínuo

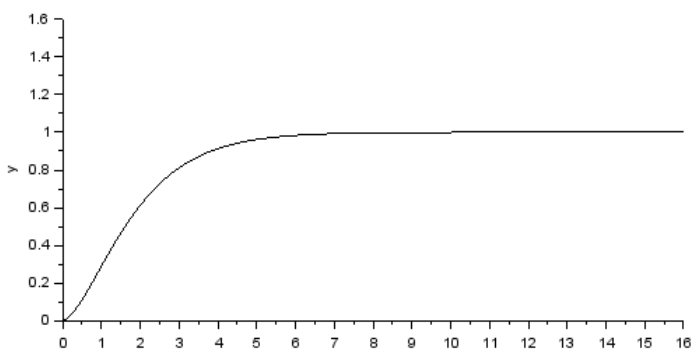

Fonte: Autores.
Figura 7 - Resposta ao degrau unitário da planta com Controlador Discreto

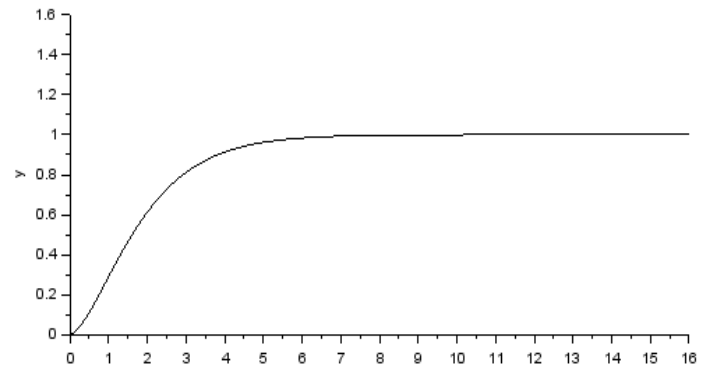

Fonte: Autores.

Com as simulações por diagramas de blocos feitas e seus resultados sendo satisfatórios, iniciou-se o projeto do sistema físico. Para tanto utilizou-se o software Proteus devido a sua interface interativa e de fácil manuseio, sendo útil tanto para simulação do sistema quanto para a sua implementação em placa de circuito impresso.

Para a realização das operações usadas nas equações do sistema utilizou-se o amplificador operacional LM324N, devido ao seu encapsulamento do tipo DIP14 possuir 4 operacionais em um único circuito integrado (CI), todos estes utilizando a mesma alimentação, contribuindo para uma menor dimensão do sistema. 
As configurações do amplificador operacional seguem equações conhecidas, a Figura 8 mostra a configuração de ganho com inversão de sinal e a Equação (31) é a lei referente a ela.

Figura 8 - Configuração de ganho com inversão de sinal.

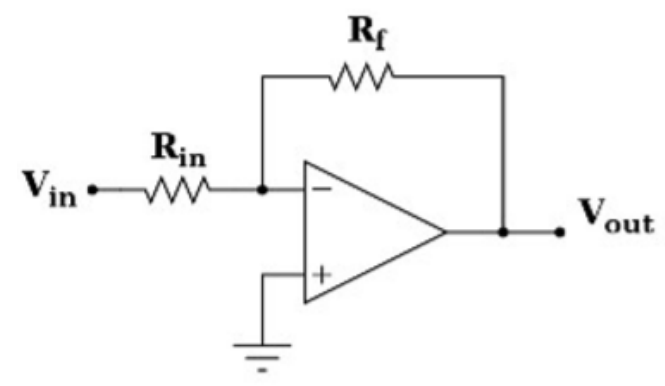

Fonte: WENDLING, 2010.

$$
V_{\text {out }}=\frac{-R_{f}}{R_{\text {in }}} \cdot V_{\text {in }}
$$

A Figura 9 mostra a configuração de soma de sinais com inversão e a Equação (32) a sua lei de funcionamento.

Figura 9 - Configuração de soma com inversão de sinal.

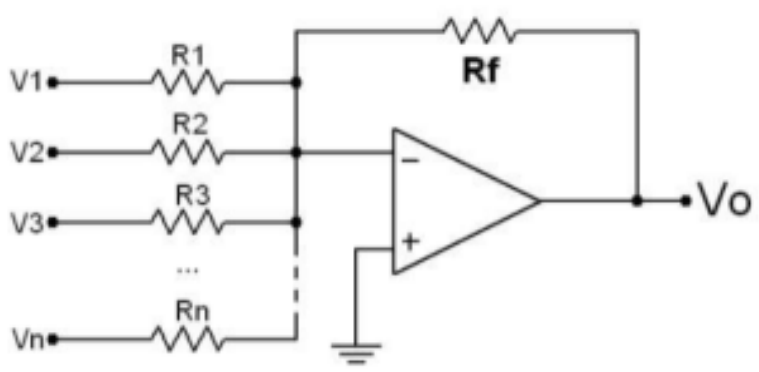

Fonte: WENDLING, 2010.

$$
V_{\text {out }}=-R_{f}\left(\frac{1}{R_{1}}+\frac{1}{R_{2}}+\cdots+\frac{1}{R_{n}}\right) \cdot V_{\text {in }}
$$

A Figura 10 mostra a configuração de integrador com inversão de sinal e a Equação (33) a sua lei de funcionamento.

Figura 10 - Configuração de integrador com inversão de sinal. 


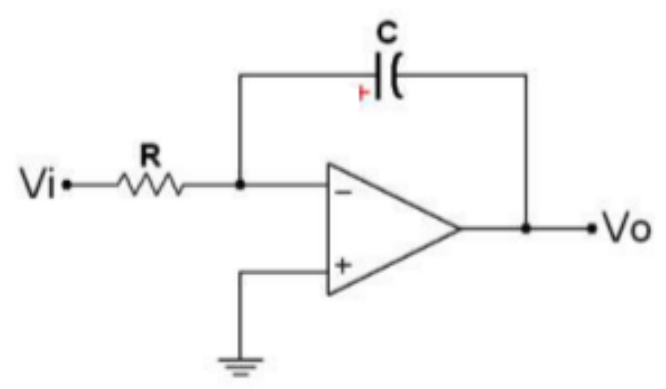

Fonte: WENDLING, 2010.

$$
\mathrm{V}_{\text {out }}=-\frac{1}{\mathrm{RC}} \int \mathrm{V}_{\text {in }} \mathrm{dt}
$$

Importante salientar que, em todas as configurações usadas há a inversão do sinal, sendo necessário utilizar um circuito de ganho unitário para adequar o sinal. Para os circuitos de ganho unitário utilizou-se resistências de 10k Ohm, que devido a sua alta impedância impossibilita que as correntes nos terminais dos circuitos integrados ultrapassem seus valores típicos. Para ganhos não unitários, utilizou-se arranjos com a resistência de $10 \mathrm{k}$ Ohm e potenciômetros lineares de $100 \mathrm{k}$, possibilitando uma ampla faixa de variação do ganho. Da mesma forma, para o circuito de soma, todas as resistências utilizadas foram de 10k Ohm, já que a intenção é de que o operacional apenas some os sinais sem acrescentar nenhum ganho. Para o integrador utilizou-se resistências de 100k Ohm e capacitores de 10u Faraday para que a se mantivesse o ganho unitário. A Figura 11 mostra o projeto do sistema no Proteus.

Figura 11 - Projeto do sistema montado com amplificadores operacionais.

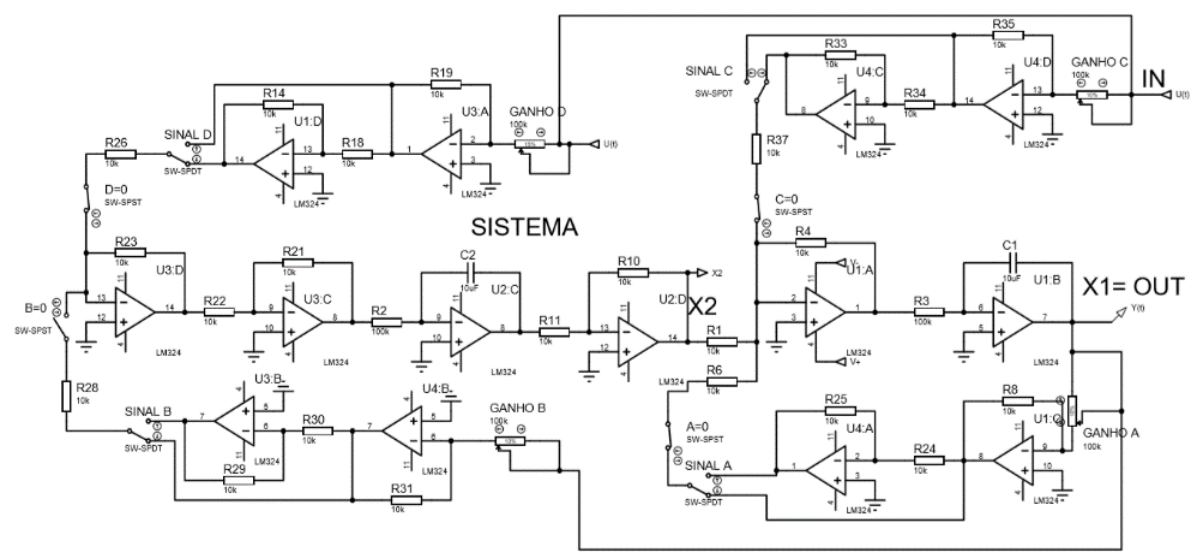

Fonte: Autores.

Aproveitando a característica das configurações utilizadas em que há inversão de sinal, utilizou-se uma chave seletora de dois pontos para definir individualmente o sinal das variáveis $a, b, c$ e $d$, de modo que se a chave estiver na primeira posição o sinal irá passar somente pelo primeiro operacional e sairá com ganho e inversão, se a chave estiver na segunda posição o sinal irá passar por um circuito de ganho unitário com inversão de sinal, ou seja, ele vai inverter o sinal de entrada. Para definir quando a variável é 0 , colocou-se uma chave simples que quando aberta, desconecta essa saída do circuito com o resto do sistema. 
(C) COBENGE 2020

"Os desafios para formar hoje o engenheiro do amanhã"
$\mathrm{Ol}$ a $\mathrm{O3}$ de dezembro Evento On-line

Para o sistema de controle utilizou-se o mesmo princípio, porém, utilizando as variáveis $\mathrm{K}_{1}, \mathrm{~K}_{2}$ e $\alpha$ como mostra a Figura 12.

Figura 12 - Projeto do sistema de controle por alocação de polos para planta do tipo 1.

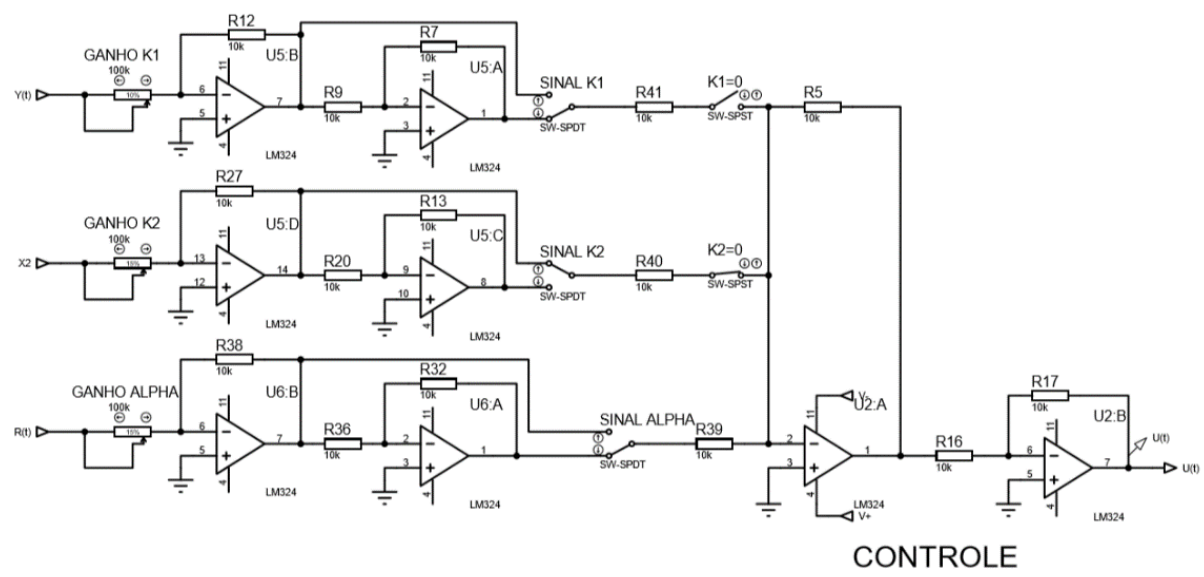

Fonte: Autores.

Após o termino do projeto via software iniciou-se a montagem do sistema em protoboard e feito os testes práticos utilizando o Arduino para enviar um sinal PWM e fazer a captação da saída do sistema.

\section{CONSIDERAÇÕES FINAIS}

As simulações no software Proteus mostraram que para essa planta, aplicando um sinal degrau unitário o sistema apresenta uma saída em forma de rampa crescente até a tensão máxima do amplificador operacional, exatamente como o resultado esperado quando feito no Xcos, ferramenta do Software Scilab. Da mesma forma a saída da planta controlada assemelha-se com o resultado da simulação do Xcos.

Partindo para o teste prático, aplicou-se um sinal degrau no sistema em malha aberta e a resposta do sistema apresentou a mesma característica das simulações anteriores. Após esse teste foi colocado o sistema de controle por arduino e por amplificador operacional e realizado os testes, o resultado assemelhou-se ao obtido na simulação via Proteus como mostra a Figura 13.

Figura 13 - Saída do sistema montado em protoboard.
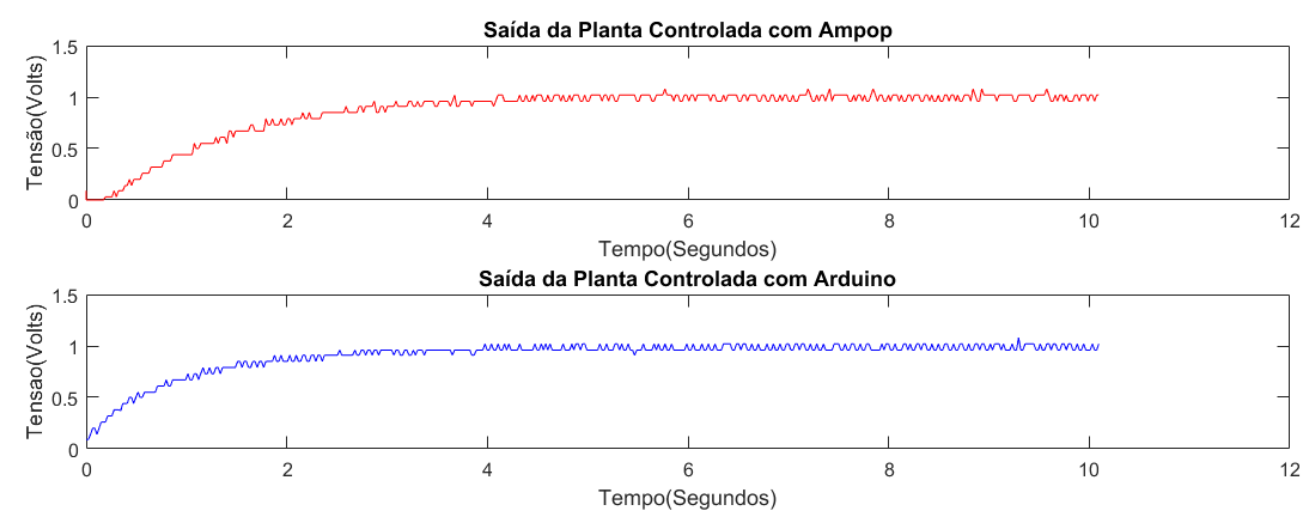
Fonte: Autores.

Após realizar os testes e verificar seu funcionamento, o projeto iria ser utilizado em turmas da disciplina de sistemas de controle, em que se estuda sobre o tema de sistemas em espaço de estados. Inicialmente, seria feito um roteiro de simulação buscando mostrar como se utiliza a ferramenta e mostrando alguns exemplos para a aplicação, após a elaboração do roteiro, seria feita uma aula teste somente com os professores das matérias para buscar ensinar todo o seu funcionamento. Posteriormente a avaliação e validação dos professores, a ferramenta iria ser aplicada a uma turma e no final seria feito um questionário com o objetivo de verificar a usabilidade da ferramenta na disciplina, a contribuição do projeto para o ensino da turma e as melhorias aplicáveis, com objetivo de avaliar a sua eficácia, entretanto, devido a pandemia de COVID-19, a avaliação tornou-se inviável, pois as atividades nas universidades foram suspensas temporariamente.

\title{
REFERÊNCIAS
}

OGATA, KATSUHIKO. Engenharia de Controle Moderno. Rio de Janeiro: Prentice Hall do Brasil, 1985.

SCILAB. Scilab tutorials. Disponível em: https://www.scilab.org/tutorials/.

Acesso em: 18 abr. 2020.

WENDLING, MARCELO. Amplificadores operacionais. Disponível em $<$ http $/ /$ www2.feg.unesp.br/Home/PaginasPessoais/ProfMarceloWendling/3---amplificadoresoperacionais-v2.0.pdf> Acesso em: 30 de abr. 2020.

\section{STATE SPACE MODULE FOR THE APPLICATION OF CONTROL TECHNIQUES FOR SECOND ORDER PLANTS}

\begin{abstract}
The work presents the design, implementation and application of a module in state space for second-order plants, with its use aimed at students as a way to help them better understand the theme. Simple and low-value components were used for easy elaboration and, therefore, democratization of the tool. That said, it is expected that the tool will contribute to the improvement of teaching in engineering, especially the disciplines focused on the area of dynamic control systems.
\end{abstract}

Keywords: Didactic Module. Teaching Learning. Control. State Space. 